\title{
Biplot Simulation of Length and Circumference of Different Body Regions of Swamp Buffalo (Bubalus bubalis)
}

\author{
I Putu Sampurna ${ }^{1, *}$, Tjokorda Sari Nindhia ${ }^{1}$, Ni Nyoman Werdi Susari ${ }^{2}$ and I Ketut Suatha ${ }^{2}$ \\ ${ }^{1}$ Laboratory of Biostatistics; ${ }^{2}$ Laboratory of Veterinary Anatomy, Faculty of Veterinary Medicine, Udayana \\ University, Bali, Indonesia
}

\begin{abstract}
Background: Biplot simulation using factor analysis with Promax kappa rotation of 90 is used to determine the coordinates of the length and body circumference dimensions of male and female mud buffalo in two-dimensional space. So that it can be known which body dimensions have fast, medium, and slow growth rates, and also to find out at what age male and female buffalo the growth rate is fast or slow.

Methods: This study used 3 male and female buffaloes aged 10,12, 14, 16, 18, and 20 months, so that the number of buffalo used was 36. The data obtained were analyzed using Factor Analysis with Promax Kappa 90 rotation. The object coordinates were the coordinates of male and female swamp buffalo aged 10,12, 14, 16, 18, and 20 months, determined based on the Analysis Factor Scores Regression. The location of the coordinates of the length and circumference dimensions and the location of the object coordinates of male and female buffaloes aged 10, 12, 14, 16, 18 , and 20 months are drawn using a biplot graph.

Conclusion: The biplot simulation results show that the body dimensions in quadrant II have the fastest growth rate, namely the length of the horns and the length of the neck, which are body dimensions that are slow to reach adult size. The dimensions of the body in quadrant I are body length, head length, upper and lower neck circumference. While the slow growth rate in quadrant IV indicates that the dimensions of the body are already growing or the growth is not optimal; namely, the length of the ears and the length of the tail have reached adult size. At the same time, the abdominal circumference and chest circumference are due to their growth not being optimal. Based on age and sex, the dimensions of male buffalo's length and body circumference are always greater than that of female buffalo from the age of 10-20 months. The positional distance between male and female buffalo age shows that the older the age, the shorter the distance; this result indicates that the growth rate slows down with increasing age.
\end{abstract}

Keywords: Biplot, buffalo, kappa 90, adult size, body regions, length, and circumference.

\section{INTRODUCTION}

The dimensional growth of an animal before reaching adulthood usually follows an exponential function with different growth rates among the many different parts of the body. The difference in growth rates between the body regions can be due to physiological demands, functional differences, and constituent components. Those body dimensions required to be functional earlier tend to grow faster than those that function later, or functional needs are required last. The body dimension consists of bone as its constituent component, developing earlier than that consisting of muscle and fat [1].

Sampurna and Suatha (2008) [2] reported that the growth of body regions in male Bali cattle starts from the neck, head, back of the body and ends at the front. Meanwhile, the body regions that develop and grow earlier are chest circumference, followed by abdominal circumference, front neck circumference, and back neck circumference. Sampurna et al., (2014) [3] show that the fastest growth in livestock occurs when they reach the point of inflection.

*Address correspondence to this author at the Laboratory of Biostatistics, Faculty of Veterinary Medicine, Udayana University, Bali, Indonesia;

Tel: 087862445838; E-mail: putu_sampurna@unud.ac.id
However, in many studies, researchers are also often faced with many dimensions of the body and presentations using allometric equations or the Power function. The resulting graph is often less attractive because it is impossible or difficult to present on a single image. Biplot demonstration using 90 kappa Promax rotation for exponential function can show the difference in the growth rate of the body dimensions into 3 quadrants in two-dimensional space. The body dimensions are in quadrant II for the dimensions of the body with a slow growth rate, quadrant I for the dimensions of the body that have a moderate growth rate, and quadrant IV for dimensions with fast growth rates. The growth rate of animal body dimensions from birth to adulthood is generally divided into two, when it grows accelerated and when it grows, it is slowed, and when the dimensions of the animal's body approach adult size. Based on the research results on biplot charts using kappa rotation of 90 , Bali cattle calves in quadrant II have a slow growth rate, those in quadrant I have moderate growth rates, and those in quadrant IV have a fast growth rate [4]. Factor analysis using Promax kappa rotation 90 on the body dimensions of Bali cattle illustrated in a two-dimensional eigenspace. The location of the coordinates can show the closeness of the relationship between body dimensions based on the growth rate. Suppose the dimensions of the body 
are at the same growth rate. In that case, the coordinate distance between the dimensions of the body will be close to zero, while the body dimensions the farther the coordinate distance is, the greater the difference between the growth rate [4]. Simulation of the power function with different constants and rates with the kappa 90 Promax rotation located in 3 quadrants, the coordinates of the power function does not depend on the constant of the power function and only depend on the rate of the power function. The coordinate location of the power function with different rates in the biplot simulation is divided into 2 dimension space and 3 quadrants; those located in quadrant I are classified as power function as moderate rate, quadrant II as fast rate, and quadrant IV as slow rate [5]. The dimensions of the body that have a fast growth rate are the dimensions of the body that have high growth potential and are still long-reaching adulthood. The body dimension where the growth rate is moderate is where the rate of growth is close to average. Body dimensions with a slow growth rate are those dimensions that have not yet the potential to grow, or the dimensions of the body have reached adult size so that the dimensions of the body have started to stop growing. So that the growth rate is slower and the growth rate is 0 (zero) if the dimensions of the body have grown cores.

Cluster analysis is a multivariate analysis that classifies the data obtained from research based on their characteristics. The similarity of characteristics is usually measured by the closeness between the objects. Furthermore, in grouping data based on objects, another multivariate analysis that can be used is a biplot analysis. The purpose of a biplot analysis is to show both rows (objects) and columns (variables) of the data matrix simultaneously in a low-dimensional (two- or three-dimensional) graph. Biplot demonstrations include variance and correlation between variables and the closeness of the relationships between objects, which will be able to identify object groupings [6].

Biplot simulations using factor analysis to draw rows (objects) and columns (variables) of matrix data available simultaneously in a low-dimensional graph are usually performed using varimax rotation. Mattjik and Sumertajaya (2002) [7] say that the variables will be described as arrows or vectors. Two variables with a positive correlation will be described as two vectors in the same direction or forming a sharp angle. Meanwhile, two variables with a negative correlation are described as two vectors in opposite directions or forming obtuse angles. Two unrelated variables will be represented as two vectors forming an angle of $90^{\circ}$ (right angle). Variables with a small variance will be represented as short vectors, while those with large variance will be represented as long vectors. Two objects with the same characteristics will be described as two points at a close position for the proximity between objects. An object with a position at the end of the arrow direction of a variable will have a value above the average.

Conversely, other objects positioned in the opposite direction of the variable will have values below the average. Meanwhile, the object with a position almost in the middle will be worth almost the same as the average value. Sampurna et al. (2013) [4] reported that the biplot simulation using the kappa 90 Promax rotation could show the closeness of the relationship between variables. The shorter the distance between variables, the greater the correlation between these variables. If the distance of 2 variables is close to zero (coincide), then the correlation will be close to 1 . At the same time, the angle between variables or vectors and the vector length cannot describe the correlation value and variance between variables.

Biplot is an exploratory data analysis method of various variables, showing in a graph the proximity between objects, the variance of variables, correlation between variables, and associations between variables and objects. In addition, biplot charts are used to illustrate the correlation between variables and objects in two-dimensional space. From the biplot analysis obtained three matrix approaches related to data, variables, and objects [8]. In principle, biplot analysis shows a plot graph of the $X$ matrix data by overlaying vectors in low-dimensional eigenvalues, usually twodimensional, representing the row vector $X$ (object) and the vector representing the column $X$ (variable). This graphic display is expected to get a description of the object, such as the closeness of the relationship between the object, the description of the variable, both active variance and correlation, and the relationship between object and variable [9].

In order to assess the relevant factors, the first selection of these steps is followed by factor rotation, which serves to provide a biplot graph that is easier to understand and gives more meaning to the location of the coordinates of objects and variables. Rotations can be orthogonal or tilt (allowing factors to be correlated). Promax rotation is an alternative to the non-orthogonal rotation calculation method, which is faster than the 
direct fulfillment method and is sometimes used for large data sets. In the practice of rotation in factor analysis, it is highly recommended to try several measures of the factor subspace stored for assessment of rotational stability [10].

This study aims to determine the growth rate of male and female buffalo's length and circumference dimensions based on their coordinates using a biplot graph simulation and determine which body dimensions and at what age experienced slow, medium, and fast growth rates.

\section{MATERIAL AND METHODS}

This research was conducted on male and female mud buffalo aged $10,12,14,16,18$, and 20 months, every 3 heads, so the number of buffalo used was $2 \times 6$ $x 3=36$ heads. Body dimensions measured were head length, neck length, body length, horn length, ear length, tail length, upper neck circumference, lower neck circumference, chest circumference, and abdominal circumference.

Factor Analysis analyzed the data obtained with Promax Kappa 90 Rotation; the coordinates of each body dimension were obtained from 2 eigenvectors with the first highest eigenroot as the $\mathrm{X}$-axis and the second-highest eigenroot as the $\mathrm{Y}$-axis. Position coordinates for each body dimension in a twodimensional space show the degree of correlation and closeness between different body dimensions. Body dimensions, namely the length and circumference dimensions of swamp buffalo with a close position, indicate an intimate relationship or the growth rate is almost the same, while the length and circumference dimensions of swamp buffalo with a further position indicate a greater difference in growth rates. The use of Kapa 90 Rotation Promax is intended to divide the position of the body dimensions into 3 different quadrants. The body's dimensions in quadrant I are for the length and circumference dimensions with moderate velocity, while the long dimensions with slower and faster growth rates are in quadrat II and quadrant IV. The object coordinates, namely the coordinates of male and female buffalo aged 10, 12, $14,16,18$, and 20 months, are determined based on the Analysis Factor Scores Regression. The score factor 1 is the $X$-axis (abscissa), and factor 2 is the $Y$ axis (ordinate). Location of coordinates of the dimensions of length and circumference of the body of the swamp buffalo and the location of the coordinates of the objects of male and female buffalo aged 10, 12, $14,16,18$, and 20 months are shown using a biplot graph. Analytical procedures were performed using the SPSS 25 program (Statistical Product and Service Solutions 25).

\section{RESULTS AND DISCUSSION}

The result of using two main components showed that $95,576 \%$ of the 10 body regions of male and female buffalo, with different growth rates, could be shown with a biplot graph in the Eigen 2 space different growth rates (Table 1).

The scree plot dimensions of the swamp buffalo body length and circumference in Figure 1 show that the Eigen root values decreased sharply from

Table 1: Total Variance Explained Dimension of Length and Circumference of Swamp Buffaloes Aged 10-20 Weeks

\begin{tabular}{|c|c|c|c|c|c|c|}
\hline \multirow{2}{*}{ Component } & \multicolumn{3}{|c|}{ Initial Eigen values } & \multicolumn{3}{c|}{ Extraction Sum of Squared Loadings } \\
\cline { 2 - 7 } & Total & $\%$ of Variance & Cumulative \% & Total & $\%$ of Variance & Cumulative \% \\
\hline \hline 1 & 9.276 & 92.756 & 92.756 & 9.276 & 92.756 & 92.756 \\
\hline 2 & 0.282 & 2.819 & 95.576 & 0.282 & & \\
\hline 3 & 0.212 & 2.119 & 97.695 & & & \\
\hline 4 & 0.115 & 1.154 & 98.849 & & & \\
\hline 5 & 0.068 & 0.679 & 99.528 & & & \\
\hline 6 & 0.026 & 0.263 & 99.791 & & & \\
\hline 7 & 0.012 & 0.122 & 99.913 & & & \\
\hline 8 & 0.005 & 0.052 & 99.966 & & & \\
\hline 10 & 0.003 & 0.026 & 99.992 & & & \\
\hline
\end{tabular}

Extraction Method: Principal Component Analysis. 


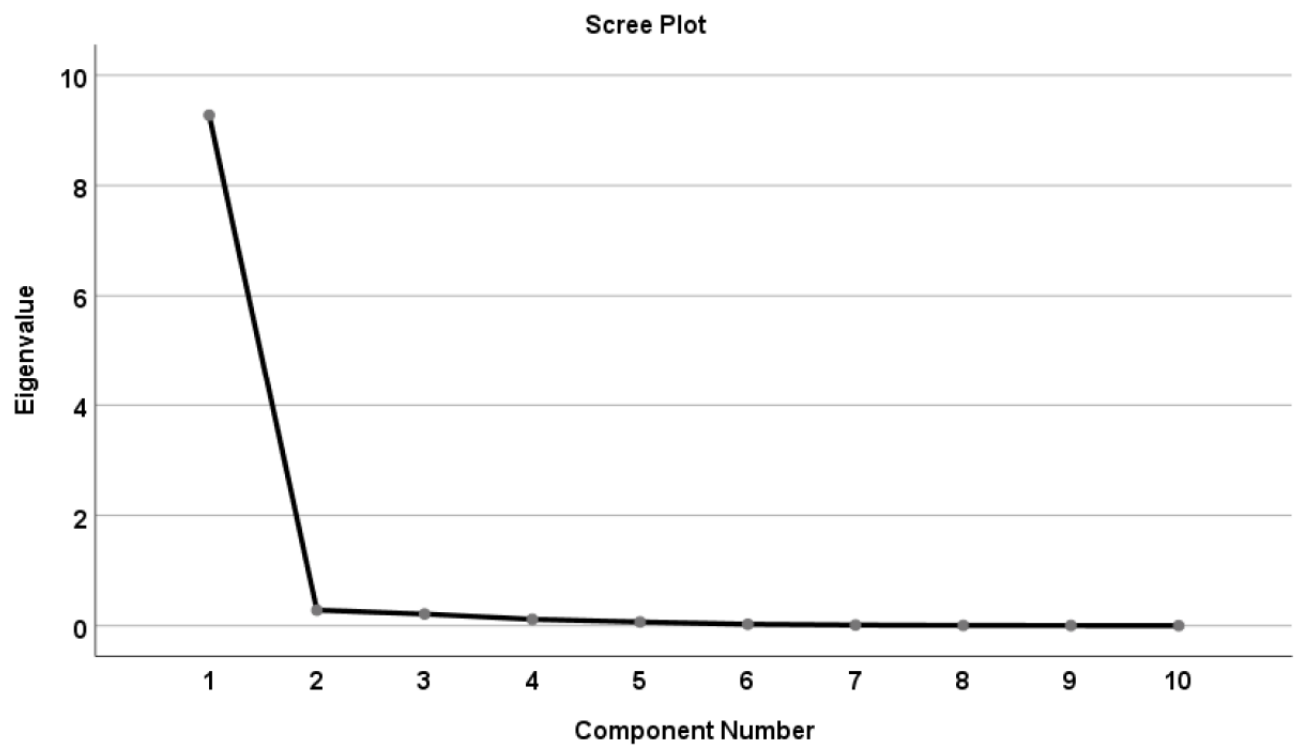

Figure 1: Scree Plot Graph of Length and Circumference of 10 -20 Month Old Swamp Buffalo.

component 1 to component 2, but no significant decrease was observed after component 2 to component 10 was shown by a flat line, so 10 body dimensions of male and female buffaloes can be shown in two-dimensional eigenvalues with component 1 as abscissa and component 2 as ordinate (Figure 1).

Location of Coordinates of the Dimensions of Length and Circumference: Based on the coordinates of component 1 as abscissa and component 2 as ordinate. Table 2 shows the dimensions of the length and circumference of the buffalo in quadrant II are neck length and horn length. In quadrant I, the dimensions are body length, head length, upper neck circumference, and lower neck circumference, and those in quadrant IV are ear length, abdominal circumference, length of tail, and bust. These results are also shown in Figure 2, a sequence of dimensions of length and circumference of square II, neck length and horn length, head length, upper neck circumference, lower neck circumference; and continues to quadrant IV, ear length, abdominal circumference, length tail and finally the chest circumference at different distances.

According to Lendhanie (2005), the age of puberty for swamp buffalo occurs at 2 - 3 years, and body maturity occurs after reaching sexual maturity. When an animal reaches adulthood, most of the organs, toes, body parts and body dimensions have reached adult size until they have stopped growing. However, some organs, toes, body parts, and body dimensions have reached the adult size or have grown nuclei before reaching sexual maturity due to the functional demands

Table 2: Coordinates of the Patten Matrix of Body Length and Circumference of Swamp Buffalo

\begin{tabular}{|c|c|c|c|}
\hline \multirow{2}{*}{ Quadrant } & \multirow{2}{*}{ Dimensions of the Length and Circumference of the Swam Buffalo } & \multicolumn{2}{|c|}{ Component } \\
\cline { 3 - 4 } & & I (Abscissa) & II (Ordinate) \\
\hline \hline \multirow{3}{*}{ I } & Neck Length & -0.59888 & 1.54251 \\
\cline { 2 - 4 } & Horn Length & -0.54459 & 1.51400 \\
\hline \multirow{3}{*}{ II } & Body_Length & 0.28942 & .68379 \\
\cline { 2 - 4 } & Head Length & 0.44899 & 0.53194 \\
\cline { 2 - 4 } & Upper Neck Circumference & 0.48310 & 0.49566 \\
\hline \multirow{3}{*}{ IV } & Lower Neck Circumference & 0.57296 & 0.41582 \\
\cline { 2 - 4 } & Ear Length & 1.01065 & -0.04847 \\
\cline { 2 - 4 } & Abdominal Circumference & 1.28356 & -0.31610 \\
\cline { 2 - 4 } & Tail Length & 1.33102 & -0.35028 \\
\cline { 2 - 4 } & Chest Circumference & 1.40309 & -0.44670 \\
\hline
\end{tabular}




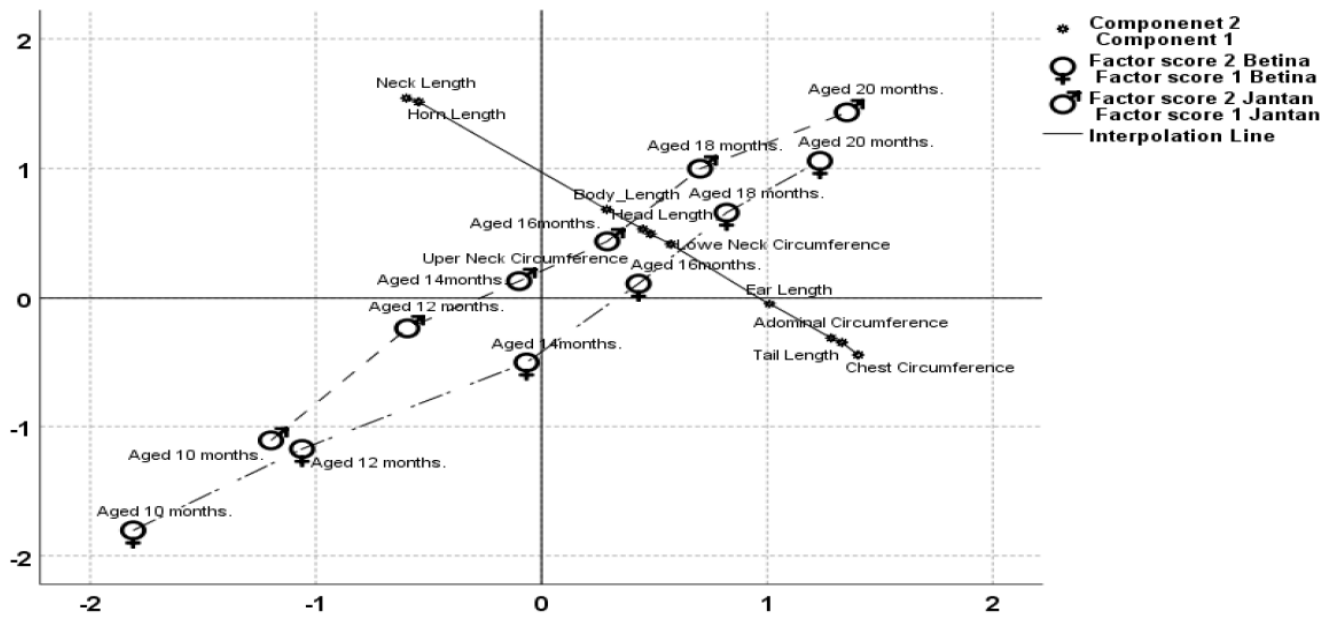

Figure 2: Biplot Graph Dimensions of Length and Circumference of Swamp Buffalo at 10 - 20 Months.

of these organs, toes, body parts, and body dimensions. The buffaloes used in this study are aged 10-20 months, have not yet reached sexual maturity, let alone adult bodies, so that the dimensions of the length and circumference of the mud buffalo have not reached the adult size. The location of the coordinates of the dimensions of length and circumference of the swamp buffalo body aged 10-20 months shows the difference in the growth rate of the length and circumference dimensions.

The results showed that the dimensions of length and circumference of swamp buffaloes reach adult size at different ages. Differences in length and circumference to reach adult size appear to be caused by differences in functional demands and constituent components of each body dimension [1, 4]. Horn length and neck length are in quadrant I, including body dimensions that reach adult size slowly, while the length of the ears and tail and the circumference of the abdomen and chest are in quadrant IV, including those who reach adult size quickly. This result is in accordance with the location of the coordinates of the power function at different rates in the biplot simulation, divided into 2-dimensional space and 3 quadrants, those located in quadrant I are classified as power functions as moderate rate or reaching moderate adult size, quadrant II as fast or slow rate reaching adult size and quadrant IV as slow or fast rate. Reach adult size the fastest [5].

Table 3 shows that the coordinates of male and female buffalo at the age of 10 and 12 are in quadrant III. while at the age of 14 months, the female buffalo is still in quadrant III, while the males are in quadrant II. After the age of 16,18 , and 20 months, the male and female buffalo coordinates are in quadrant I. The location of the coordinates based on the object, sex,

Table 3: Coordinates of Sex and Dimensions of Body Length and Circumference of Swamp Buffalo

\begin{tabular}{|c|c|c|c|c|}
\hline \multirow{2}{*}{ Sex } & \multirow{2}{*}{ Age } & \multirow{2}{*}{ Quadrant } & \multicolumn{2}{|c|}{ Component } \\
\hline & & & I (Abscissa) & II (Ordinate) \\
\hline \multirow{4}{*}{ Male } & 10 & III & -1.19927 & -1.10764 \\
\hline & 14 & II & -0.09947 & 0.13139 \\
\hline & 16 & I & 0.29093 & 0.43816 \\
\hline & 20 & I & 1.35340 & 1.43308 \\
\hline \multirow{4}{*}{ Female } & 10 & III & -1.81012 & -1.80399 \\
\hline & 12 & III & -1.06116 & -1.17378 \\
\hline & 14 & III & -0.06635 & -0.50479 \\
\hline & 16 & 1 & 0.43005 & 0.11171 \\
\hline
\end{tabular}


and age shows the size of the dimensions of the length and circumference of the swamp buffalo.

The location of the object coordinates based on sex and age of swamp buffalo (Figure 2) shows that close to the $X$-axis, the size is near average (below the $X$ axis), which is in quadrant III. This shows that the length and circumference dimensions are still below the average, which is above the $\mathrm{X}$-axis. While in quadrants II and I, the dimensions of the length and circumference are above the average. The younger the age of the buffalo, the smaller the length and circle dimensions, the farther the coordinates are below the $X$ axis, conversely the older the age, the larger the length and circle dimensions, the farther the coordinates are above the $\mathrm{X}$ axis [7].

Biplot Simulation at Dimensions of Length and Circumference: The position of the coordinates farthest below the $\mathrm{X}$-axis is a female buffalo aged 10 months, then followed by a male buffalo aged 10 months and a female buffalo aged 12 months, male buffalo aged 12 months, and female buffalo aged 14 months the coordinates are still under the $\mathrm{X}$-axis. The coordinates of the 14-month-old male buffalo and the 16-month-old female have started to be above the X-axis, then the older the location of the coordinate is, the farther above the $\mathrm{X}$-axis different. The older male and female buffalo have closer coordinate positions; this shows that the older the age is, the slower the speed because the closer to reaching adult size. Differences in position coordinate distance and proximity between ages can, in turn, identify groupings between ages or among objects [8]. This is in accordance with previous research by Sampurna et al., (2013) [4] that the fastest growth rate of animal body dimensions occurs when it reaches the inflection point, and at that age, the position of the coordinates is the longest.

The coordinate position at the same age shows that male buffalo is always above female buffaloes; this result shows that the overall dimensions of the length and circumference of male swamp buffalo are always more significant than that of female swamp buffaloes.

\section{CONCLUSION}

Biplot simulation using Promax Kapa 90 rotation can be used to show differences in the growth rate of the length and circumference dimensions of male and female mud buffaloes aged $10-20$ months; and can also be used to show the dimensions of the length and body circumference of mud buffalo, which have slow, medium, and high growth rates. Based on gender and age, the rotation of the Promax Kapa 90 biplot can also show at what age the dimensions of the body length and circumference of the mud buffalo are below average and above average.

\section{REFERENCES}

[1] Swatland HJ. Structure and Development of Meat Animals Prentice-Halllnc., Englewood Cliff, New Jersey Stephen 1984.

[2] Sampurna IP, Suatha IK. The Allometric growth of Length and Circumference Body Dimension of Bali cattle Males. Journal of Veterinary 2008; 9: ISSN: 1411-8327, Higher Education Accreditation No. 55 / DIKTI / Kep / 2005. Page I: 41-44.

[3] Sampurna IP, Saka IK, Oka GL, Sentana P.. Patterns of Growth of Bali Cattle Body Dimensions. ARPN Journal of Science and Tecnology 2014; 4(1).

[4] Sampurna IP, Saka IK, Oka IGL, Sentana P. Biplot Simulation of Exponential Function to Determine Body Dimensions'Growth Rate of Bali Calf. Canadian Journal on Computing in Mathematics Natural Sciences Engineering and Medicine 2013; Vol. 4. No. ISSN: 1923-1660.

[5] Sampurna IP, Nindhia TS. Biplot Simulation of Power Function to Determine Growth Rate of Animal. Indian Journal Applied Research 2017; 7(6): ISSN 2249-555X IF: 4.894 | IC Value : 79.96

[6] Ariawan MA, Kencana IPEN, Suciptawati BLP. Comparison Analysis Clump (Cluster) and Biplot in Grouping. E-Jurnal Matematika 2013; 2: 17-22. https://doi.org/10.24843/MTK.2013.v02.i04.p053

[7] Mattjik AA, Sumertajaya M. Applications Multiple Variable Analysis. Bogor Exercise books SPSS Statistics 2002.

[8] Steven R. Advanced linear algebra. Graduate Texts in Mathematics, New York. 10. Goodness of fit of biplots and correspondence analysis. Biometrika 2008; 89: 423-436. https://doi.org/10.1093/biomet/89.2.423

[9] Rinaldo AA. Structural Equation Modeling Approach PLS and SEM. Faculty of Science and the Graduate Program UB 2008.

[10] Abdi H. Factor Rotations in Factor Analyses. Program in Cognition and Neurosciences, MS: Gr.4.1, The University of Texas at Dallas, USA. Landhanie, UU. 2005. Karakteristik Reproduksi Kerbau Rawa Dalam Kondisi Lingkungan Peternakan Rakyat. Bioscientiae, 2003; 2(1): http://bioscientiae.tripod.com.

https://doi.org/10.6000/1927-520X.2021.10.09

(c) 2021 Sampurna et al.; Licensee Lifescience Global.

This is an open access article licensed under the terms of the Creative Commons Attribution Non-Commercial License (http://creativecommons.org/licenses/by-nc/3.0/) which permits unrestricted, non-commercial use, distribution and reproduction in any medium, provided the work is properly cited. 